\title{
Automatic Gun Targeting System using Face Detection, IR and Ultra Sonic Sensor
}

\author{
Aditya Prasad, Jayant Gupta, Yogesh Sharma, M. Jasmine Pemeena Priyadarsini
}

\begin{abstract}
In this paper, our objective is to devise a new defence mechanism using automation, which will seek to provide a viable solution to tighten the security around the border areas and simultaneously reduce the burden on soldiers. With the help of automation, we can increase the efficiency of safeguarding our borders in remote areas to a great extent, and this will also result in a better quality of surveillance due to this technological intervention. The main focus for us will be in the creation of an active and real-time device for the armed forces, which will help safeguard the relatively remote border areas where human mobility is restricted. The devised mechanism will consist of three modules: face detection of a human intruder, wireless communication, and hardware detection of an intruder. The automation system in this defence mechanism majorly consists of face detection of a human intruder, which is supported and complemented by Ultrasonic and IR Sensors. This system is implemented using Arduino microcontroller, which will be complemented with NodeMCU to provide necessary wireless communication for sending intruder detection and location information to the cloud, which will trigger the gun to shoot down the enemy. The use of wireless communication over GSM is preferred solely because the control stations receive Wi-Fi signals directly from the satellite, which is very reliable and fast. The main application of our research work is to ensure safeguarding border region around remote areas where patrolling is difficult for army personnel and will be implemented by the armed forces.
\end{abstract}

Keywords: face detection, human intruder, wireless communication, cloud.

\section{INTRODUCTION}

This paper focuses on the defense application of auto-target detection using Python with the help of Arduino-UNO microcontroller and sensor base automated gun firing system, which targets the living object within the range of the sensor. There is an application of human face detection, which helps in identifying human intruders and commanding to shoot them with the gun firing system. Face detection will eliminate the risk

Revised Manuscript Received on May 25, 2020.

* Correspondence Author

Aditya Prasad*, School of Electronics Engineering, Vellore Institute of Technology, Vellore, Tamil Nadu, India. E-mail: adityaprsd14@gmail.com

Jayant Gupta, School of Electronics Engineering, Vellore Institute of Technology, Vellore, Tamil Nadu, India.Email: guptajayant20@gmail.com

Yogesh Sharma, School of Electronics Engineering, Vellore Institute of Technology, Vellore, Tamil Nadu, India. E-mail: yogeshsharma10128@gmail.com

Dr. M. Jasmine Pemeena Priyadarsini, Higher Academic Grade, School of Electronics Engineering, Vellore Institute of Technology, Vellore, Tamil Nadu, India E-mail: jasmin@vit.ac.in

(C) The Authors. Published by Blue Eyes Intelligence Engineering and Sciences Publication (BEIESP). This is an open access article under the CC BY-NC-ND license (http://creativecommons.org/licenses/by-nc-nd/4.0/) of hunting animals and will increase efficiency. The camera captures the image of the human intruder. The face detection of The automatic gun firing is primarily based on IR sensors, Ultrasonic sensors, and face detection using Arduino Uno microcontroller and transmitting and receiving unit with the firing gun. The transmission and reception of sensor information is done with the help of NodeMCU, which also updates the information in the cloud accessible to the control stations. Information regarding image detection of the human intruder gets updated in the cloud along with the image of the intruder sent to the control station via an E-mail alerting the army personnel. This paper is designed to help in adding an extra edge in making any country's defense system combative and sharp in accordance with the present and future threats to the border areas of any country from undesirable intruders. This device further can be enhanced in detecting the friendly and enemy forces and also can be used in any better future applications, which may stand as a guardian for prolonged peace and sovereignty of humanity.

\section{COMPONENTS REQUIRED}

\section{A. Infrared Sensor}

The intruder of any form will emit heat and will be in motion. Thus, an electronic IR sensor "Infrared Sensor" has been interfaced with the system to determine the presence of an intruder. The infrared wavelength range is $850 \mathrm{~nm}$.

\section{B. Node MCU}

NodeMCU is used in wireless communication for transmission and reception of information regarding the presence of intruders at the border areas, and it is interfaced with the microcontroller. It is a low-cost open-source IoT platform that is compatible with Arduino UNO microcontroller providing wireless communication. The term "NodeMCU" properly speaking refers to the firmware instead of the associated development kits.

\section{Ultrasonic Sensor}

The ultrasonic sensor works on the principle of reflected signals similar to radar and sonar systems. It measures the time between sending a message and receiving an echo from which it calculates the distance at which the object is located. Thus, using the application, it will detect the presence of an intruder in remote areas of the border.

\section{Liquid Crystal Display}

LCD is used to display the presence of an intruder, which will help to alert the army personnel and take the necessary actions. It will also demonstrate that a human intruder's face is detected

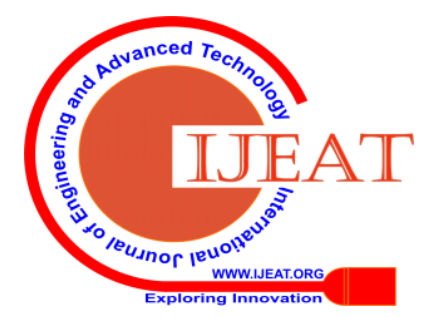


along with firing at the intruder.

\section{E. Arduino-Uno R3 microcontroller development board}

This microcontroller is used to process the various inputs provided by the sensors, which is then interfaced with Python to detect human face and, in turn, update the control station about intruders using the cloud. This development board has an inbuilt ATMEGA 328 microcontroller.

\section{F. Adafruit IO}

Adafruit IO is used for a cloud application. It majorly deals with uploading data that has been acquired from the sensors to the cloud. Moreover, it has integrated feed sharing along with fine-tuned privacy controls, which allows as well as restricts access to the data.

\section{FACE DETECTION}

\section{A. Haar Cascade Classifier Algorithm}

The classifier is based on the Haar Wavelet technique, which is used to analyze pixels by putting the image acquired into squares by function. It is optimal for live face detection and includes four significant processes, namely Haar feature selection, creation of basic image acquired during the live stream, Adaboost training, and cascade classifiers. The Haar feature selection mainly distinguishes the features that are unique to a human face. Adaboost is simply a training technique that helps in selecting only required features by splitting positives from negatives. Lastly, Cascading classifiers are used to reduce computational time and increase the detection rate.

\section{B. Pseudocode}

The presence of any humans is detected in the targeted area by real-time Adafruit IO acquired by the camera using PYTHON.

Adafruit IO files are included along with OpenCV, OS, SMTPlib, SSL, and e-mail packages, which are present in MIME (Multipurpose Internet Mail Extensions). Then SMTPlib is used to send the E-mail from source to destination via server. Then a string variable is declared where a value is stored, then we store ADAFRUIT IO KEY and USERNAME in variables and then call it in a function to enable cloud in the code. Then we load the Haar cascade classifier, which is the primary algorithm used for face detection and capture the video using open $\mathrm{CV}$ function. After this, we define a function for sending an E-mail. We describe all the parameters of E-mail that is to be posted and subsequently attach the image along with it. An exception of error is created just in case if there's no internet connectivity indicating that the E-mail cannot be sent. Then the image captured gets converted into grayscale and detects the face, then highlights the face by a circle when the camera recognizes it. The image of the face is then sent through e-mail along with 'face detected' on the python output shell. The variable which was initially created is passed through the cloud function, which sends the data onto the cloud.

\section{Hardware Pseudocode}

Initially, header files of LCD and I2C are included, and after that, pins are assigned to different sensors as well as other components. Then the function is defined where IR sensor and echoPin of Ultrasonic sensor are set as input and
trigPin along with LED as output. LCD prints 'BORDER AREA' when Arduino is started. Then a function is defined for the working of Ultra Sonic sensor, which includes a formula according to which the sensor will calculate the distance of the object from it. These calculations will be displayed on the serial monitor in Arduino IDE. If the reach of the object from the sensor is less than 20 units, then it will turn on the LED and print 'GUN FIRING' on LCD, or else it'll continue to be in off state. A function for IR sensor is also defined simultaneously where if low; then the LCD will display 'GUN FIRING' and LED will turn on or else it'll continue to be in off state. Lastly, a function is defined for face detection where when it reads a byte from I2C serial communication, LCD will display 'INTRUDER DETECTED' and LED will turn on or else it will continue to be off.

\section{TABLES, FIGURES AND ABBREVIATIONS}

\section{A. Abbreviations and Acronyms}

(i) LCD: Liquid Crystal Display.

(ii) LED: Light Emitting Diode.

(iii) I2C: Inter IC. This is a multi-master bus; multiple chips can be connected to the same bus and each one is capable of acting as a master by initiating a transfer.

(iv) MIME: Multipurpose Internet Mail Extensions.

(v) OS: Operating System

(vi) SMTPlib: Simple Mail Transfer Protocol; it handles sending and routing e-mail between mail servers. Python provides SMTPlib module and in turn it defines an SMTP client session object.

(vii) SSL: Secure socket layers. It's a networking protocol designed for securing connections web clients and web servers over the internet.

(viii) OpenCV: Open Source Computer Vision Library. Library of programming functions which deals with real time computer vision.

\section{B. Figures and Tables}

I. Pin Configuration Table:

Table- I: ARDUINO PIN CONFIGURATION

\begin{tabular}{|c|c|}
\hline COMPONENT & PIN NUMBER \\
\hline \multirow{2}{*}{$\begin{array}{l}\text { ULTRASONIC } \\
\text { SENSOR }\end{array}$} & TRIGGER PIN - 6 \\
\hline & $\begin{array}{ll}\text { ECHO PIN } & -5\end{array}$ \\
\hline IR SENSOR & 4 \\
\hline LED & 7 \\
\hline \multirow[t]{5}{*}{ LCD } & RESET -13 \\
\hline & ENABLE - 12 \\
\hline & D4 -11 \\
\hline & D5 $\quad-10$ \\
\hline & D6 $\quad-9$ \\
\hline \multicolumn{2}{|c|}{$\begin{array}{l}\text { Published By: } \\
\text { Blue Eyes Intelligence Engineering } \\
\text { \& Sciences Publication } \\
\text { (C) Copvriaht: All riahts reserved. }\end{array}$} \\
\hline
\end{tabular}




\section{open?access}

\begin{tabular}{|l|l|}
\hline \multirow{2}{*}{ Node MCU } & D7 - 8 \\
& GPIO1(SDA)- A4 \\
\cline { 2 - 2 } & GPIO2(SCL)- A5 \\
\hline
\end{tabular}

II. Flowchart

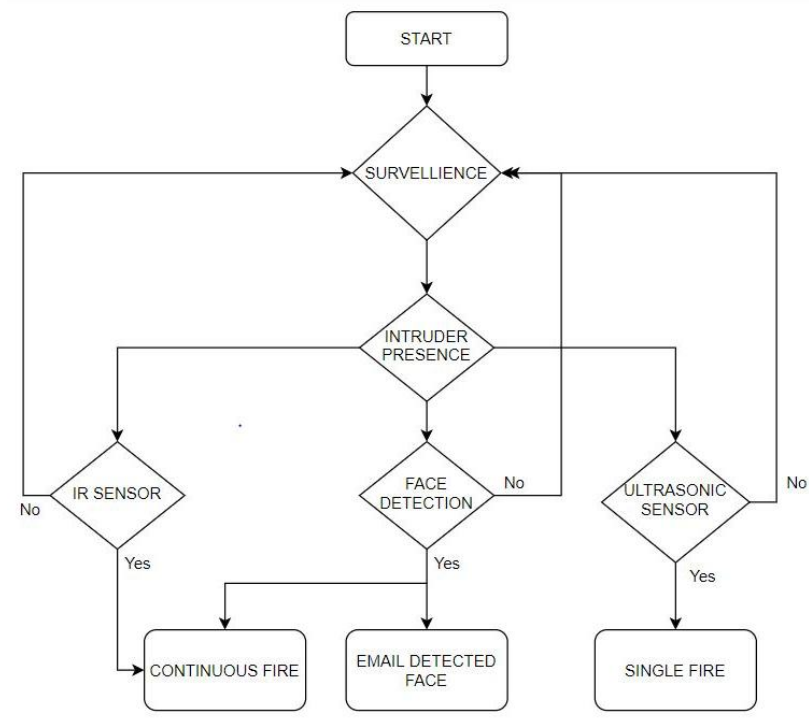

Figure 1. Flow Chart.

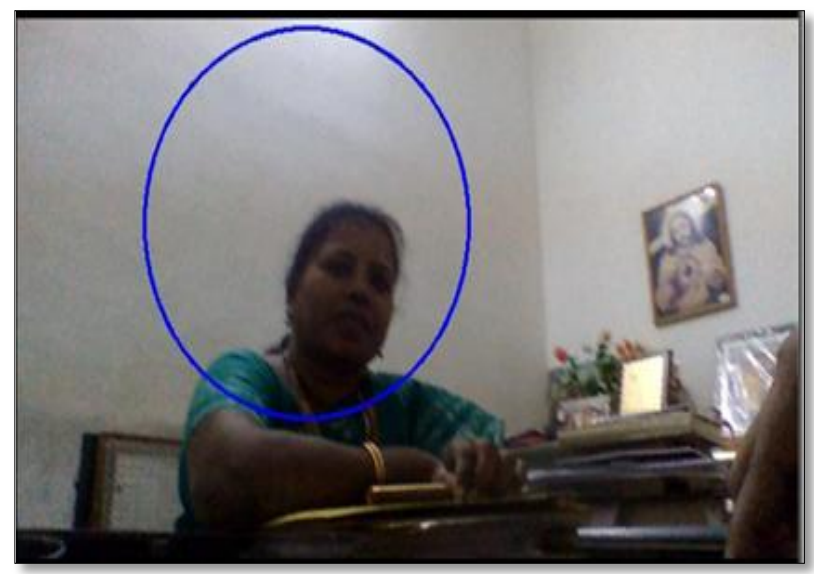

Figure 2. Detected Face 1

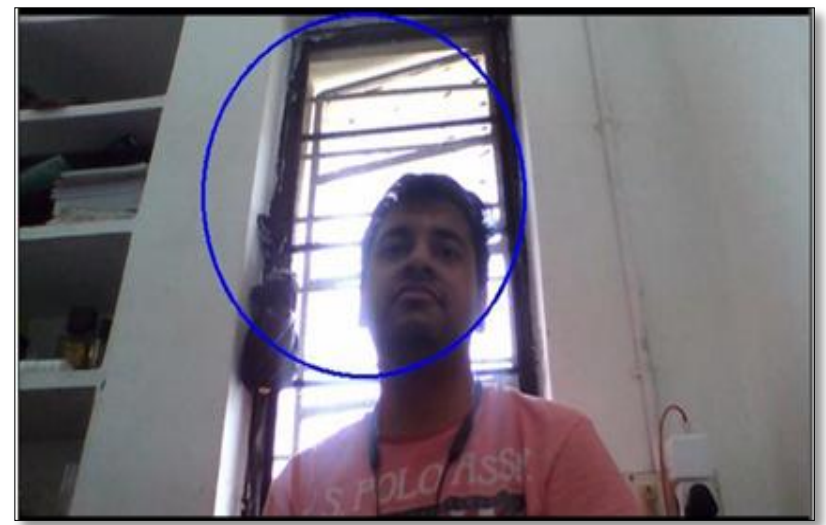

Figure 3. Detected Face 2

\section{OBSERVATION}

A. Cloud

Published By:

Blue Eyes Intelligence Engineering

\& Sciences Publication

(c) Copyright: All rights reserved.
Adafruit IO is a real-time application cloud that is used to monitor the camera. It enables the user to define many different devices to be connected. In Figure 4, we see the cloud server named $\mathrm{POONCH}$, which gives details like date and time along with the message that the intruder has been detected. There can be many servers which can be defined as per the coverage area requirement.

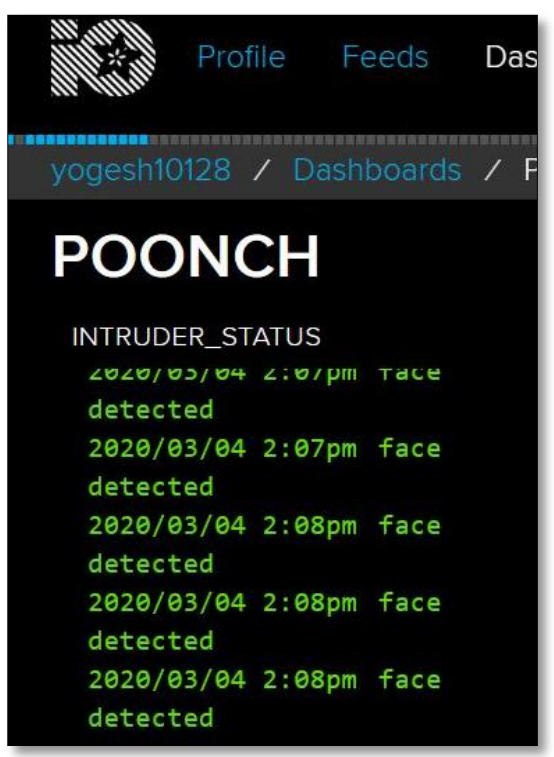

Figure 4. Adafruit cloud output

B. LCD

In Figure 5, the LCD has been powered and initialized. During the initialization of LCD, the BORDER_AREA message has been displayed. In Figure 6 , it is seen that wherever an intruder is detected or any sensor gives back, and a positive acknowledgment INTRUDER_DETECTED message has been displayed.

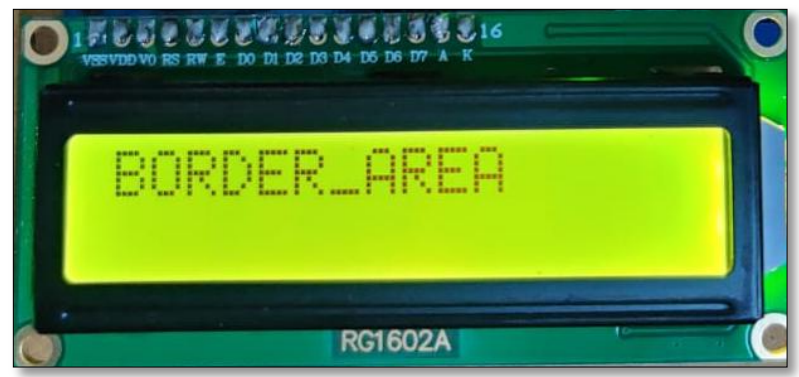

Figure 5. LCD Initialization

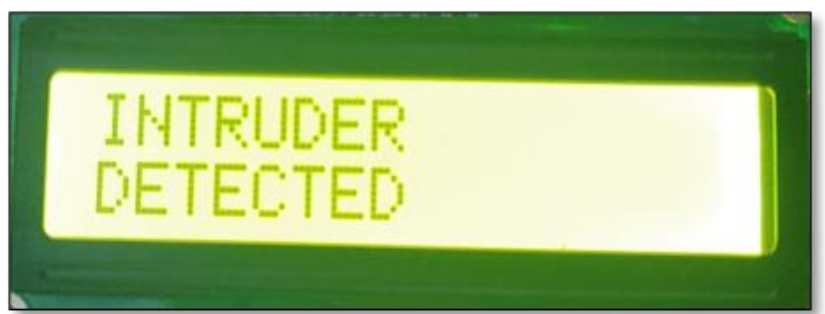

Figure 6. LCD intruder detected via Face Detection Algorithm 


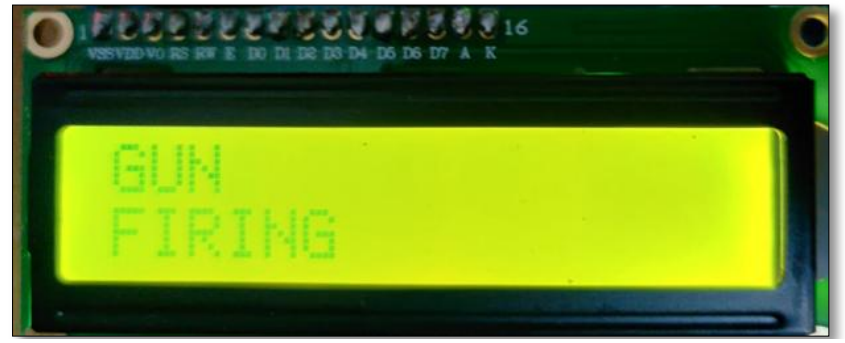

Figure 7. LCD on Intruder Detection due to Sensor

\section{Serial Monitor}

In the Arduino microcontroller, we can see the serial monitor to be giving the following reading given in Figure 7. Whenever there is 0, i.e., the Ultrasonic sensor has given positive acknowledgment, and the gun would start firing.

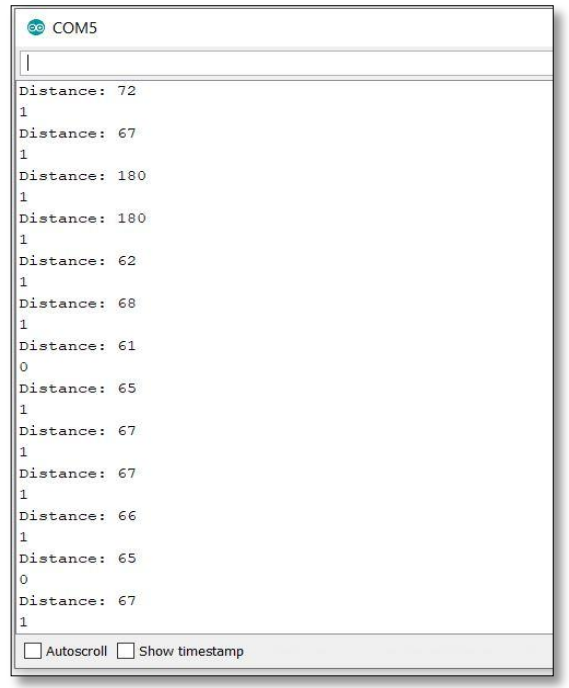

Figure 8. Serial Monitor

\section{E-mail Output}

In Figure 7, there is an e-mail alert message which is provided by the cloud to know who the intruder is. The camera captures the image and processes it into the cloud, and the copy is further sent to e-mail to keep the record of the intruder.

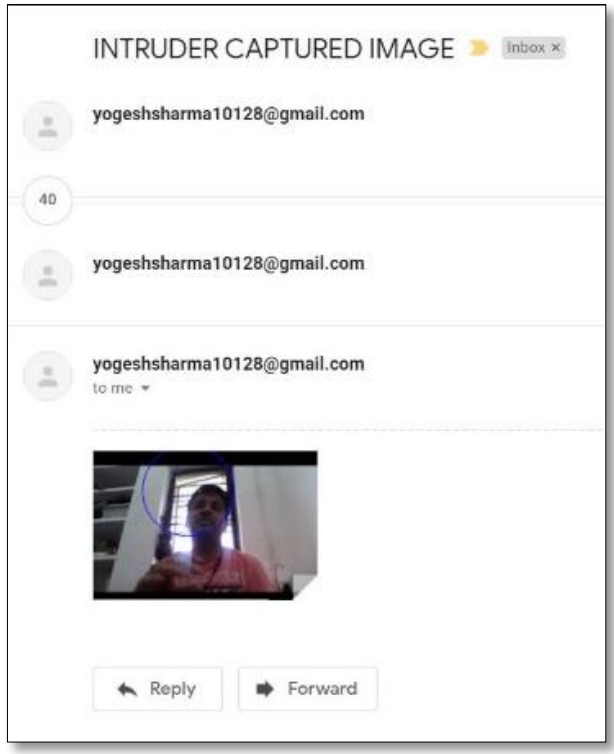

Figure 9. E-mail received

\section{RESULT AND DISCUSSION}

The application of an Automatic gun targeting system using Face Detection is providing accurate data. The image of the intruder, as detected by the camera, is sent to E-Mail, which easily identifies the person. The project is further complemented by two sensors, i.e., IR and Ultrasonic Sensor. These sensors help additional security by acting as a backup in case of camera failure. In case the intruder can escape from the camera, these sensors help in close range for his identification.

Haar Cascade's face detection algorithm enables one to detect multiple faces at the same instant. As long as the camera gun detects the face, it would continuously fire, and simultaneously the image of the intruder will be sent to the e-mail.

Adafruit IO cloud is used to determine which gun has been triggered and at what instance. This cloud enables us to find precisely where there is intrusion detected by any hardware. One can remotely monitor all the guns from a distant place.

When the intruder's presence triggers the IR sensor, their world is a single blink in the LED, showing that a single shot has been fired. Any external agent can trigger an IR sensor; hence, a single shot fire is commanded by the microcontroller.

When the Ultrasonic sensor acknowledges the presence of an intruder, LED glows for a longer duration of time, showing that multiple shots have been fired.

\section{CONCLUSION}

The project works on three different parts, which include IR, Ultrasonic sensor, and face detection. The e-mail notification, with the picture of intruder upon face detection, is sent to the individual mail id, which in this case, will be addressed to the person in charge of the control station. The gun will continuously fire as long as there is a human face detected. When the IR sensor gets the reading, there is continuous firing, and a display of gun firing is made on LCD. When the Ultrasonic sensor detects the movement, there is a single shot fired along with the LCD. The live report on the Adafruit cloud from the face detected from the camera is also obtained. All of the attributes mentioned above of the defense mechanism would ensure that there is continuous surveillance of the remote areas, making it difficult to patrol for soldiers. Locating and targeting an intruder will become more accurate and efficient. This will result in an overall improvement in security around the border areas, especially in those regions which are difficult to access for soldiers. There will be a sharp improvement in resource deployment, logistics, and other related costs of patrolling our extended border areas. The efficiency improvement will help the armed forces to deploy the resources in other focus areas both in terms of defense personnel and cost-saving. Hence, the primary objectives of our research are achieved through the devised mechanism.

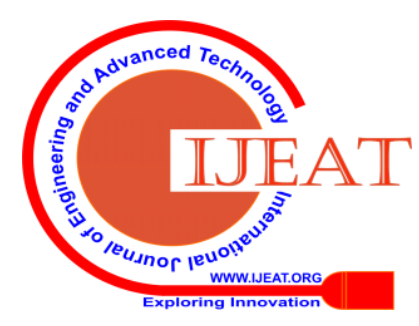




\section{FUTURE SCOPE}

The project can further be improved by using an advanced high-resolution camera that supports night vision or thermal vision. The study can also be done using this system for airborne targets. The study will focus more on targeting very fast-moving targets like airplanes. Further research can be done to design a system that can track multiple targets. The survey can even be extended to allow the system to track multiple targets with different target criteria. Instead of using a simple microcontroller like Arduino, the project can be further be extended by interfacing with an advanced microcontroller.

\section{ACKNOWLEDGMENT}

We are grateful to School of Electronics Engineering VIT University Vellore and our guide Dr. M. JASMINE PEMEENA PRIYADARSINI, Higher Academic Grade, VIT University, Vellore, Tamil Nadu for guiding and assisting us in completing the project.

\section{REFERENCES}

1. N. Djelal, N. Mechat, and S. Nadia, "Target tracking by visual servoing," in Systems, Signals and Devices (SSD), 2011 8th International Multi-Conference on. IEEE, 2011, pp. 1-6.

2. N. Djelal, N. Saadia, and A. Ramdane-Cherif, "Target tracking based on surf and image based visual servoing," in Communications, Computing and Control Applications (CCCA), 2012 2nd International Conference on. IEEE, 2012, pp. 1-5.

3. E. Iflachah, D. Purnomo, and I. A. Sulistijono, "Coil gun turret control using a camera," EEPIS Final Project, 2011.

4. A. M. Idris, K. Hudha, Z. A. Kadir, and N. H. Amer, "Development of target tracking control of gun-turret system," in Control Conference (ASCC), 2015 10th Asian. IEEE, 2015, pp. 1-5.

5. J. D. S. Munadi and M. F. Luthfa, "Fuzzy logic control application for the prototype of gun-turret system (arsu 57-mm) using matlab," 2014.

6. T. M. Nasyir, B. Pramujati, H. Nurhadi, and E. Pitowarno, "Control simulation of an automatic turret gun based on force control method," in Intelligent Autonomous Agents, Networks and Systems (INAGENTSYS), 2014 IEEE International Conference on. IEEE, 2014 pp. 13-18.

7. G. Ferreira, "Stereo vision-based target tracking for a gun turret utilizing low performance components," Ph.D. dissertation, University of Johannesburg, 2006.

8. H. D. B. Brauer, "Real-time target tracking for a gun-turret using low cost visual servoing," Master's thesis,University of Johannesburg,

9. Ö. Gümü say, "Intelligent stabilization control of turret subsystems under disturbances from unstructured terrain," Ph.D. dissertation, Middle East Technical University, 2006.

10. Y. LeCun, Y. Bengio, and G. Hinton, "Deep learning," Nature, vol.

11. A. Krizhevsky, I. Sutskever, and G. E. Hinton, "Imagenet classification with deep convolutional neural networks," in Advances in neural information processing systems, 2012, pp. 1097-1105.

12. K. Simonyan and A. Zisserman, "Very deep convolutional networks for large-scale image recognition," arXiv preprint arXiv:1409.1556, 2014.

13. C. Farabet, C. Couprie, L. Najman, and Y. LeCun, "Learning hierarchical features for scene labeling," IEEE transactions on pattern analysis and machine intelligence, vol. 35, no. 8, pp. 1915-1929, 2013.

14. A. Graves, M. Liwicki, S. Fernández, R. Bertolami, H. Bunke, and J. Schmidhuber, "A novel connectionist system for unconstrained handwriting recognition,"IEEE transactions on pattern analysis and machine intelligence, vol. 31, no. 5, pp. 855-868, 2009.

15. A.-r. Mohamed, G. E. Dahl, and G. Hinton, "Acoustic modeling using deep belief networks," IEEE Transactions on Audio, Speech, and Language Processing, vol. 20, no. 1, pp. 14-22, 2012.

16. R. Girshick, "Fast r-cnn," in Proceedings of the IEEE International Conference on Computer Vision, 2015, pp. 1440-1448.

17. R. Girshick, J. Donahue, T. Darrell, and J. Malik, "Rich feature 2006. [Online]. 521, no. 7553, pp. 436-444, 2015. hierarchies for accurate object detection and semantic segmentation,"

in Proceedings of the IEEE conference on computer vision and pattern recognition, 2014, pp. 580-587.

\section{AUTHORS PROFILE}

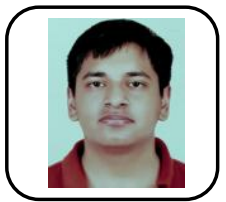

Aditya Prasad has pursued his education from Delhi Public School, Vasant Kunj, New Delhi from 2002-2010 after which he resumed his education in Nairobi, Kenya for two and a half years. There he studied in Braeburn International School. He returned to Delhi Public School, Vasant Kunj, New Delhi in 2013 and completed his higher secondary education there. Delhi Public School, Vasant Kunj was affiliated CBSE curriculum and Braeburn International School was affiliated with IGCSE curriculum. He is currently pursuing under-graduation in B.Tech from Vellore Institute Of Technology, Vellore, Tamil Nadu. He is doing major in Electronics and Communications Engineering. He started pursuing this degree from 2016 and will be completing it in 2020. He has done a few internships and trainings in his respective field. He has done training conducted by Technex affiliated with IIT-BHU in robotics and automation in 2017. He did internship in Real Estate Financial Management in the field of IoT in 2018 from Ericsson. His last internship was from Solarflare company which specializes in VLSI field in 2019. He has done a project on Street Light Automation using 8051 microcontrollers with the help of Keil IDE.

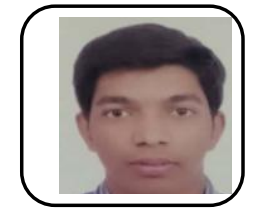

Jayant Gupta has completed his schooling from Delhi Public School, Greater Noida, Uttar Pradesh, which comes under the CBSE curriculum. He is currently pursuing his B-Tech in Electronics and Communication Engineering at the Vellore Institute of Technology, Vellore, Tamil Nadu. He has attended a Robotics and Automation workshop conducted by Technex at Delhi Technological University, which is affiliated with IIT BHU in the year 2017. He did an internship from Nanotrics Innovation Pvt ltd where he worked with Nexys DDR4 Artix 7 microcontroller in the year 2018. He did an internship in Solarflare Communication, where he worked in the VLSI department in 2019. He has received music scholar award from his school. He used to be part of the National Service Scheme for two years during my graduation from 2017-2019. He did many projects out of which Density Based Traffic Control System and Automatic Street Light System are some.

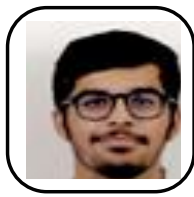

Yogesh Sharma has pursued his education from Maheshwari Public School, Jawahar Nagar, Jaipur from 2004-2016. He was the sports captain of his school and have played volleyball at national level and Athletics at state level. He is currently pursuing under-graduation in B. Tech from Vellore Institute of Technology, Vellore, Tamil Nadu. He is doing major in Electronics and Communications Engineering. He started pursuing this degree from 2016 and will be completing it in 2020. He has done his internship in Bhartiya Sanchar Nigam Limited (BSNL). He was a part of social service camp of 7 days with National Service Scheme (NSS). He has done a project on Street Light Automation using 8051 microcontrollers with the help of Keil IDE and automatic railway gate controller using same technology, and currently doing his final year project on Automatic Gun Targeting System using Face Detection, IR and Ultra Sonic Sensor.

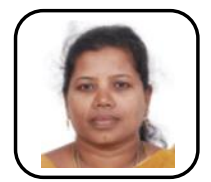

Dr. M. Jasmine Pemeena Priyadarsini obtained B.E. degree from Madras University in 1992 and M.E. degrees from Madurai Kamaraj University, Madurai in 1995. She earned his Ph.D. from Vellore Institute of Technology, Vellore, INDIA in 2014. She has published more than 45 research papers in National and International journals and reputed conferences. She has a teaching experience of about 25 years in Vellore Institute of Technology, Vellore in India. Presently, she is serving as Professor at Vellore Institute of Technology, India. She is a life member of Indian Society for Technical Education, IEEE society Membership, Fellow of Institution of Engineers, Fellow of Institution Electronics and Telecommunication Engineers. She has authored about four technical books. His research areas include Digital Image Processing, Digital signal processing, Optical Signal Processing, Light wave Communication Systems, Optical Coding Theory and Biometric Image Processing. She is a reviewer of several international conferences and journals.

Eyes Intelligence Engineering 